\title{
Determinantes de incapacidade temporária para o trabalho de longa duração - Estudo InCIT
}

Joana Costa Gomes, ${ }^{1,2}$ Alexandra Sousa, ${ }^{3}$ Ana Isabel Ribeiro, ${ }^{3}$ Filipa Silva, ${ }^{4}$ Mara Galhardo, ${ }^{4}$ Sofia Esquível, ${ }^{1}$ Teresa Maia Fernandes ${ }^{5}$

\begin{abstract}
RESUMO
Objetivos: Este trabalho pretende avaliar determinantes sociodemográficos, laborais e patológicos de ausência laboral prolongada. Tipo de estudo: Caso-controlo prospetivo

Local: Unidade Local de Saúde de Matosinhos

População: Foram considerados casos indivíduos entre os 18 e os 65 anos que se encontravam com Certificado de Incapacidade Temporária (CIT) há pelo menos 30 dias por doença natural. Os controlos foram selecionados aleatoriamente, emparelhados por médico de família.

Métodos: Recolheu-se informação relativa a variáveis sociodemográficas e patológicas (cirurgia recente, patologia psiquiátrica, patologia osteoarticular e doença oncológica) e aplicaram-se duas escalas (APGAR Familiar e a Escala de Satisfação no Trabalho). Calcularam-se Odds Ratio (OR) ajustados para género, idade e escolaridade e respetivos Intervalos de Confiança (IC) a 95\% para estimar a associação entre estes determinantes e possibilidade de estar com CIT prolongado.

Resultados: Durante o período de estudo foi emitido CIT prolongado a 76 indivíduos, para os quais foram aleatoriamente selecionados 76 controlos. Cerca de $56 \%$ dos casos e $62 \%$ dos controlos eram homens, sem diferenças significativas entre géneros. A idade média dos casos foi superior à dos controlos $(50,0$ versus 42,$5 ; \mathrm{p}<0,001)$ e os casos eram em geral menos escolarizados $(p<0,001)$. Constatou-se que mais casos exerciam profissões de colarinho azul $(p=0,001)$ e obtiveram pontuações mais baixas no APGAR Familiar $(p=0,008)$. Verificou-se ainda um aumento da possibilidade de CIT prolongado na presença de patologia osteoarticular (OR 6,14; IC $95 \%$ 2,79-13,48), oncológica (OR 4,62; IC 95\% 1,18-18,08) e psiquiátrica (OR 5,21; IC $95 \% 2,14-12,70$ ) e cirurgia recente (OR 14,49 ; IC $95 \%$ 3,83 - 54,80).

Conclusões: Este trabalho poderá auxiliar os médicos de família a identificar os indivíduos mais vulneráveis ao absentismo laboral prolongado, para permitir implementação de medidas preventivas custo-efetivas que permitam reduzir o impacto pessoal, familiar e social e os custos que lhe estão associados.
\end{abstract}

Palavras-chave: Absentismo Laboral; Fatores Epidemiológicos; Estudo de Casos e Controlos.

\section{INTRODUÇÃO}

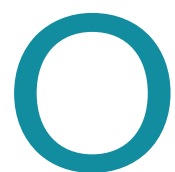

absentismo laboral é um importante problema de saúde pública, associando-se a custos diretos e indiretos não desprezíveis, parecendo existir associação entre a incapacidade laboral prolongada e determinadas carate-

\footnotetext{
${ }^{1}$ Interna de Medicina Geral e Familiar, USF Lagoa, ULS Matosinhos ${ }^{2}$ Instituto de Saúde Pública da Universidade de Porto

${ }^{3}$ Interna de Medicina Geral e Familiar, USF Oceanos, ULS Matosinhos

${ }^{4}$ Interna de Medicina Geral e Familiar, USF Dunas, ULS Matosinhos

${ }^{5}$ Interna de Medicina Geral e Familiar, USF Infesta, ULS Matosinhos
}

rísticas individuais, sociais e laborais..$^{1-3} \mathrm{O}$ conhecimento das associações entre fatores demográficos e ambiente laboral na população ativa é importante para o desenvolvimento de estratégias com vista à redução do absentismo laboral prolongado, principalmente tendo em conta o contexto socioeconómico atual, em que a otimização dos recursos assume um papel fundamental. ${ }^{4,5}$

Estudos anteriores demonstraram que a incapacidade laboral prolongada é mais frequente no género feminino, ${ }^{1,6} \mathrm{em}$ trabalhadores de «colarinho azul $»^{3,7} \mathrm{e}$ in- 
divíduos de nível socioeconómico inferior, ${ }^{7}$ estando também descrita a associação entre patologia músculo-esquelética e ausência laboral. ${ }^{4} \mathrm{Um}$ conjunto de fatores psicológicos e fatores relacionados com a profissão contribuem também para o absentismo laboral, nomeadamente baixa satisfação profissional, elevadas expectativas, conflitos profissionais, entre outros. ${ }^{4,8} \mathrm{Em}$ muitos países ocidentais a patologia músculo-esquelética é a principal causa de absentismo laboral, seguida da patologia psiquiátrica ${ }^{2}$ mas, na população portuguesa, existe pouca evidência relativa às ausências ao trabalho e seus determinantes. ${ }^{3}$

Em Portugal a incapacidade laboral é certificada em impresso próprio denominado certificado de incapacidade temporária (CIT) emitido pelo médico assistente. ${ }^{9}$ Com base na legislação em vigor é considerada CIT de longa duração um CIT com duração superior a 30 dias (artigo n. ${ }^{\circ} 296$ do Código do Trabalho).

Uma vez que o combate ao absentismo laboral passa pela sua prevenção, é necessário um conhecimento aprofundado das suas causas e relações. ${ }^{8}$ A hipótese que subjaz a este trabalho é que os indivíduos com CIT $>30$ dias têm diferentes características sociodemográficas e patológicas que os indivíduos sem CIT > 30 dias. Pretendemos estudar esta hipótese avaliando variáveis sociodemográficas e presença de patologia psiquiátrica, osteoarticular, oncológica e cirurgia recente em indivíduos com e sem incapacidade para o trabalho. Será também avaliada a influência da presença de problemas familiares e laborais.

\section{MÉTODOS}

Realizou-se um estudo caso-controlo em 4 unidades de saúde familiar (USF) do ACES de Matosinhos (USF Oceanos, USF Dunas, USF Infesta e USF Lagoa), onde casos de indivíduos com CIT com duração superior a 30 dias foram comparados com controlos.

Foram selecionados para os casos indivíduos com idade superior a 18 anos e inferior a 65 anos que se encontravam com CIT há mais de 30 dias por doença natural das listas de orientadores de formação dos investigadores (sete médicos de família) - cujas CIT foram renovadas pelos médicos de família ou pelos investigadores nos meses de janeiro, fevereiro e março de 2012. Para cada caso foi escolhido um controlo da mesma lista de utentes após aleatorização a partir da lista ordenada de números de identificação (NOP) dos indivíduos com idades compreendidas entre os 18 e os 65 anos de cada lista. Foram excluídos, grávidas e reformados. Caso o indivíduo selecionado aleatoriamente não fosse elegível para o estudo era selecionado o indivíduo imediatamente a seguir.

Foram estudadas as variáveis sociodemográficas: género, idade, escolaridade, tipo de trabalho (colarinho branco ou colarinho azul) e condição perante o trabalho (trabalhador por conta própria; trabalhador por conta de outrem - setor público, trabalhador por conta de outrem - setor privado; desempregado; outra), estado civil (solteiro; casado/união de facto; viúvo; divorciado/separado), tipo de família a que pertence o indivíduo (nuclear, alargada, unitária, monoparental, reconstruída, outro) e patologia/situação clínica/ /problema (cirurgia recente, patologia psiquiátrica, patologia osteoarticular e doença oncológica). Foi considerada cirurgia recente aquela que tinha ocorrido nos 6 meses anteriores, tendo sido considerados os seguintes tipos de cirurgia: geral, ortopédica, ginecológica ou outra. Foi ainda obtida informação relativa à presença ou não de patologia psiquiátrica, oncológica ou osteoarticular. Foram considerados os códigos ICPC2 P01 a P06, P70 a P86, P98 e P99 para patologia psiquiátrica; A79, B72-74, D74-77, F74, H75, K72, L71, N74, R84/85, S77, T71/73, U75-77/79, W72, X75-77 e Y77/78 para patologia oncológica e L01 a L29 e L70 a L99 para patologia osteoarticular, registados no processo do indivíduo, sendo esta informação completada com a informação obtida durante a entrevista telefónica e com dados do processo físico.

Foram ainda aplicadas duas escalas, APGAR familiar de Smilkstein, versão validada para a língua portuguesa, ${ }^{10}$ e Escala de Satisfação do Trabalho (EST) ${ }^{11}$, para avaliação de problemas familiares e laborais, respetivamente. A escala EST (Siqueira M, 1995) é um instrumento multidimensional, construído com o objetivo de avaliar o grau de satisfação do trabalhador relativamente a cinco dimensões do seu trabalho: satisfação com o salário (três itens), satisfação com os colegas de trabalho (três itens), satisfação com a chefia (três itens), satisfação com as promoções (três itens) e satisfação com a natureza do trabalho (três itens). Trata-se de um questionário com perguntas de escolha múltipla, usando uma escala tipo Likert, com sete categorias de res- 
postas para cada item, que vão de «totalmente insatisfeito» (1) a «totalmente satisfeito» (7). A EST permite calcular cinco scores médios (um para cada dimensão) e um score geral que engloba todas as dimensões. Neste trabalho foi apenas calculado o scoregeral de satisfação. Para o cálculo do score geral somam-se os valores assinalados pelos respondentes e divide-se esse valor pelo número total de questões do questionário (15 questões). O resultado ficará entre 1 e $7 .{ }^{11}$ Uma vez que a escala de satisfação no trabalho utilizada foi traduzida para português, mas validada numa população brasileira, foi realizado um teste piloto com 20 indivíduos para avaliar a sua aplicabilidade, que permitiu verificar a ausência de dificuldades da compreensão da escala.

A colheita de dados de casos e controlos foi feita por entrevista telefónica realizada em abril de 2012, pelos investigadores. Estes foram previamente treinados para que existisse uniformização das entrevistas, minimizando o potencial viés de entrevistador. Foram efetuadas até três tentativas de contacto telefónico após as quais foram considerados não-respondentes. No início do contacto foi explicado o objetivo do estudo e obtido consentimento informado oral. Para aplicação de duas escalas que requerem autopreenchimento foram oferecidas ao indivíduo três hipóteses: deslocar-se ao centro de saúde para preenchimento em momento oportuno, envio das escalas por correio ou envio das escalas por correio eletrónico. Na altura da entrevista foi preenchida uma folha de dados, construída no programa Google Forms ${ }^{\circledR}$, que permite codificação automática em base de dados. A base de dados guardada em Google Docs ${ }^{\circledR}$ foi protegida por palavra passe do conhecimento exclusivo dos investigadores e em nenhum dos registos foram identificados os utentes, de forma a garantir confidencialidade. Durante a entrevista foram colocadas questões diretamente ao utente que foram complementados com os dados disponíveis no processo clínico informático (códigos ICPC2 - International Classification of Primary Care, second edition) e físico do caso/controlo.

O cálculo do tamanho amostral foi realizado utilizando o menu Statcalc do programa Epi info ${ }^{\circledR}$ versão 6 (1993) para IC (Intervalo de Confiança) 95\% e poder $80 \%$, assumindo uma prevalência de exposição nos não expostos de $50 \%$ e um OR (Odds Ratio) esperado de 3.0 o que correspondeu a 65 casos e 65 controlos. O OR es- perado foi obtido com base na revisão da evidência anterior, tendo em conta o OR médio encontrado para a associação ente as diversas variáveis independentes utilizadas e a ausência laboral prolongada. ${ }^{12-14}$

A análise dos dados foi realizada procedendo à descrição comparativa de casos e controlos usando métodos de estatística descritiva. Foram calculados OR ajustados para género, idade e escolaridade e respetivos IC a 95\% utilizando regressão logística para estimar a associação entre determinantes e possibilidade de CIT prolongado. Foi usado um nível de significância estatística de 0,05 . Todos os cálculos foram conduzidos utilizando o software STATA ${ }^{\circledR}$, Version 11.2 (StataCorporation, College Station, Texas).

\section{CONSIDERAÇÕES ÉTICAS}

O protocolo foi aprovado pela comissão de ética da Unidade Local de Saúde de Matosinhos (ULSM). Foi obtido consentimento informado oral para participação no estudo a todos os indivíduos (casos e controlos) no início da entrevista telefónica realizada para recolha de dados. A base de dados foi restrita, através de palavra-passe, a consulta pelos investigadores e a todos os indivíduos foi atribuído um código, não passível de identificação para garantir confidencialidade.

\section{RESULTADOS}

Entre janeiro e março de 2012 foram identificados nas sete listas de médicos de família 76 casos de CIT superior a 30 dias de duração para os quais foram selecionados aleatoriamente 76 controlos, emparelhados por lista de utentes.

Verificou-se que os casos eram significativamente mais velhos (idade média em anos 50,0 vs 42,5, $p<$ $0,001)$ e menos escolarizados $(47,4 \%$ dos casos versus $14,5 \%$ dos controlos com escolaridade entre 0 e 4 anos, $p<0,001)$ que os controlos. Não se registaram diferenças significativas entre casos e controlos em relação ao género ou ao estado civil. Em relação à atividade laboral, não se registaram diferenças na condição perante o trabalho. Verificou-se, no entanto, que $82,7 \%$ dos casos eram profissionais de colarinho azul em comparação com apenas $57,4 \%$ dos controlos $(p=0,001)$. Houve uma tendência para APGAR familiar mais baixo nos casos do que nos controlos $(p=0,008)$. Relativamente ao tipo de família não foi encontrada qualquer asso- 
ciação. Finalmente, quanto à presença de patologia verificou-se que os casos tinham mais cirurgias nos últimos seis meses, em comparação com os controlos $(p<0,001)$ sem diferenças no que respeita ao tipo de cirurgia. O mesmo se verificou em relação à presença de patologia osteoarticular $(p<0,001)$, oncológica $(p<0,001)$ ou psiquiátrica ( $p<$ 0,001) (Quadro I).

Não se verificou associação estatisticamente significativa entre o género, estado civil, condição perante o trabalho, tipo de trabalho, agregado familiar ou APGAR familiar e CIT prolongado. Em relação a idade, embora de forma não significativa, os OR mostram tendência para aumento de possibilidade de CIT com aumento da idade. Níveis de escolaridade mais elevados associaram-se significativamente a menor possibilidade de CIT prolongado. Indivíduos satisfeitos, segundo a escala EST, com a sua atividade laboral têm menos possibilidade de usufruir de CIT prolongado quando comparados com indivíduos insatisfeitos (OR 0,34; IC 95\% 0,12 a 1,00).

\begin{tabular}{|c|c|c|c|}
\hline & $\begin{array}{c}\text { Casos } \\
(n=76)\end{array}$ & $\begin{array}{l}\text { Controlos } \\
(n=76)\end{array}$ & $p$ \\
\hline \multicolumn{4}{|l|}{ Género [n(\%)] } \\
\hline Masculino & $43(56,6)$ & $47(61,8)$ & \multirow[t]{2}{*}{0,509} \\
\hline Feminino & $33(43,4)$ & $29(38,2)$ & \\
\hline Idade (média \pm dp) & $50,0( \pm 9,89)$ & $42,5( \pm 9,79)$ & $<0,001$ \\
\hline \multicolumn{4}{|l|}{ Idade (anos) [n(\%)] } \\
\hline $18-30$ & $4(5,2)$ & $11(14,5)$ & \multirow[t]{3}{*}{$<0,001$} \\
\hline $31-50$ & $28(36,8)$ & $45(59,2)$ & \\
\hline $51-65$ & $44(58,0)$ & $20(26,3)$ & \\
\hline \multicolumn{4}{|l|}{ Estado civil [n(\%)] } \\
\hline Solteiro & $7(9,2)$ & $9(11,8)$ & \multirow[t]{4}{*}{0,766} \\
\hline Casado/união de facto & $57(75,0)$ & $59(77,6)$ & \\
\hline Viúvo & $2(2,6)$ & $1(1,3)$ & \\
\hline Divorciado/Separado & $10(13,2)$ & $7(9,2)$ & \\
\hline \multicolumn{4}{|l|}{ Escolaridade (anos) [n(\%)] } \\
\hline $0-4$ & $36(47,4)$ & $11(14,5)$ & \multirow[t]{5}{*}{$<0,001$} \\
\hline $5-6$ & $13(17,1)$ & $18(23,7)$ & \\
\hline $7-9$ & $18(23,7)$ & $16(21,0)$ & \\
\hline $10-12$ & $7(9,2)$ & $18(23,7)$ & \\
\hline$>12$ & $2(2,6)$ & $13(17,1)$ & \\
\hline \multicolumn{4}{|l|}{ Condição perante o trabalho [n(\%)] } \\
\hline Trabalhador por conta própria & $14(18,4)$ & $8(10,5)$ & \multirow[t]{5}{*}{0,053} \\
\hline Trabalhador por conta de outrem - setor público & $7(9,2)$ & $18(23,7)$ & \\
\hline Trabalhador por conta de outrem - setor privado & $43(56,6)$ & $33(43,4)$ & \\
\hline Desempregado & $12(15,8)$ & $16(21,1)$ & \\
\hline Outra & $0(0,0)$ & $1(1,3)$ & \\
\hline \multicolumn{4}{|l|}{ Tipo de trabalho $[\mathrm{n}(\%)]^{*}$} \\
\hline Colarinho branco & $13(17,3)$ & $32(42,7)$ & \multirow[t]{2}{*}{0,001} \\
\hline Colarinho azul & $62(82,7)$ & $43(57,3)$ & \\
\hline \multicolumn{4}{|l|}{ Escala de satisfação com o trabalho $[\mathrm{n}(\%)] \dagger$} \\
\hline Insatisfação & $17(29,8)$ & $10(16,4)$ & \multirow[t]{3}{*}{0,219} \\
\hline Indiferença & $14(24,6)$ & $17(27,9)$ & \\
\hline Satisfação & $26(45,6)$ & $34(55,7)$ & \\
\hline \multicolumn{4}{|l|}{ Tipo de família [n(\%)] } \\
\hline Nuclear & $49(64,5)$ & $58(77,3)$ & \multirow[t]{6}{*}{0,356} \\
\hline Alargada & $15(11,7)$ & $11(13,3)$ & \\
\hline Unitária & $6(7,9)$ & $3(4,0)$ & \\
\hline Monoparental & $4(5,3)$ & $3(4,0)$ & \\
\hline Reconstruída & $2(2,6)$ & $0(0,0)$ & \\
\hline Outro & $0(0,0)$ & $1(1,3)$ & \\
\hline \multicolumn{4}{|l|}{ APGAR familiar [n(\%)]" } \\
\hline $0-3$ & $5(6,8)$ & $0(0,0)$ & \multirow[t]{3}{*}{0,008} \\
\hline $4-6$ & $10(13,5)$ & $3(4,1)$ & \\
\hline 7-10 & $59(79,7)$ & $71(95,9)$ & \\
\hline
\end{tabular}




\begin{tabular}{|l|r|r|r|}
\hline QUADRO I. (Continuação) & \multicolumn{1}{|c|}{} \\
& \multicolumn{1}{|c|}{$\begin{array}{c}\text { Casos } \\
(\mathbf{n = 7 6 )}\end{array}$} & $\begin{array}{r}\text { Controlos } \\
(\mathbf{n = 7 6 )}\end{array}$ & $\boldsymbol{P}$ \\
\hline Cirurgia recente [n(\%)] & $29(38,2)$ & $3(4,0)$ & $<0,001$ \\
Tipo de cirurgia [n(\%)] & $7(25,0)$ & $2(66,7)$ & 0,134 \\
$\quad$ Cirurgia geral & $9(32,2)$ & $0(0,0)$ & \\
$\quad$ Cirurgia ortopédica & $2(7,1)$ & $1(33,3)$ & \\
$\quad$ Cirurgia ginecológica & $10(35,7)$ & $0(0,0)$ & \\
$\quad$ Outra & $55(72,4)$ & $19(25,0)$ & $<0,001$ \\
\hline Patologia osteoarticular [n(\%)] & $18(23,7)$ & $3(4,0)$ & $<0,001$ \\
\hline Patologia oncológica [n(\%)] & $33(43,4)$ & $11(14,5)$ & $<0,001$ \\
\hline Patologia psiquiátrica [n(\%)] & & & \\
\hline
\end{tabular}

*4 sem dados; † 34 sem dados; " 3 sem dados

Indivíduos que foram submetidos a cirurgia nos últimos seis meses (OR 14,49; IC 95\% 3,83 a 54,80) e indivíduos com patologia osteoarticular (OR 6,14; IC 95\% 2,79 a 13,48), oncológica (OR 4,62; IC 95\% 1,18 a 18,08) ou psiquiátrica (OR 5,21; IC 95\% 2,14 a 12,70) tem possibilidade significativamente maior de permanecerem ausentes do trabalho por período superior a 30 dias (Quadro II). De referir que a associação entre CIT prolongada e a presença de patologia osteoarticular foi independente do tipo de trabalho (colarinho branco $v s$ colarinho azul; OR ajustado para género, idade, escolaridade e tipo de trabalho 6,10; IC 95\% 2,72 a 13,67).

\section{DISCUSSÃO}

De acordo com os resultados obtidos neste estudo, os indivíduos mais velhos, menos escolarizados e com patologia musculosquelética, oncológica, psiquiátrica ou história de cirurgia nos últimos seis meses têm maior possibilidade de se encontrarem com CIT prolongado.

A evidência anterior mostra que os aspetos laborais, nomeadamente a baixa satisfação profissional, elevadas expectativas e conflitos profissionais têm vindo a ser relacionados com a incapacidade laboral para o trabalho. ${ }^{2,4,8}$ Também o tipo de trabalho parece relacionar-se com o grau de absentismo laboral, sendo que este é tipicamente superior nos trabalhadores de "colarinho azul". ${ }^{3,7}$ Os nossos resultados vêm de encontro a estes

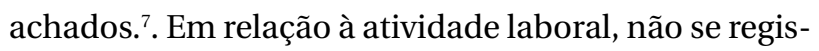
taram, no presente estudo, diferenças na condição pe- rante o trabalho. Este facto vem de encontro aos dados da literatura que identificam fatores de absentismo nos trabalhadores dependentes similares aos dos trabalhadores independentes. ${ }^{5}$ De ressalvar ainda que, de acordo com o código do trabalho, tanto trabalhadores por conta de outrem como trabalhadores independentes têm direito a usufruir do CIT, embora no caso dos trabalhadores independentes o subsídio só seja atribuído após os primeiros 30 dias (Decreto-Lei $n .^{\circ}$ 28/2004, de 4 de fevereiro na redação dada pelos Decretos-Lei $n .^{\circ}$ 146/2005, de 26 de agosto e n. ${ }^{\circ}$ 302/2009, de 22 de outubro - Regime jurídico de proteção na doença). Em relação aos desempregados, apesar de o subsídio por doença não poder ser acumulado com o subsídio de desemprego, um indivíduo inscrito no centro de emprego tem que justificar as suas ausências por doença também através de um CIT. Estas particularidades provavelmente influenciaram os resultados obtidos neste estudo quando comparados com a realidade de outros países.

Apesar do presente estudo não ter encontrado associação estatisticamente significativa com o género, em estudos anteriores o género feminino tem vindo a ser relacionado com o absentismo laboral. ${ }^{1,3}$ As diferenças entre géneros são justificadas pelas diferenças biológicas, o efeito de género e fatores socioculturais. Todavia, verifica-se também que, nos países e nos grupos etários em que as mulheres são significativamente mais faltosas ao trabalho, a ausência laboral parece ser explicada maioritariamente por problemas de saúde relacionados com a gravidez ou o cataménio e que as ausências são geralmente por períodos curtos. ${ }^{1}$ As diferenças entre o presente estudo e a evidência anterior poderão ser explicadas pelo fato do presente estudo ter excluído absentismo por gravidez de risco e ter focado a sua atenção nas ausências laborais prolongadas. Não obs- 
tante, as diferenças interculturais e legislativas devem ser tidas em conta para a explicação do fenómeno da ausência laboral nas mulheres. ${ }^{1}$

Os resultados obtidos mostram que a população mais velha tem maior possibilidade de absentismo laboral prolongado, o que confirma a tendência habitualmente encontrada na literatura. ${ }^{3}$ Estes dados poderão resultar do declínio na capacidade física, bem como do aumento das comorbilidades associado à idade. ${ }^{12}$

A incapacidade prolongada para o trabalho parece também ser superior nos indivíduos com história pessoal de patologia psiquiátrica, tal como verificado em estudos anteriores. ${ }^{2,6,15}$ Trata-se de um fenómeno de importância crescente, transversal a toda a Europa Ocidental e que parece ser influenciado por fatores geográficos mas também pelo género feminino, tipo de doença mental, aumento da idade e menor rendimento económico. ${ }^{6,16} \mathrm{O}$ risco de marginalização e de incapacidade permanente destes doentes não é desprezível, pelo que a prevenção e o tratamento destes doentes são fundamentais. ${ }^{6}$ A prevenção deve incidir também em intervenções que visem melhorar o ambiente laboral, na medida em que um mau ambiente laboral pode propiciar o aparecimento de patologia psiquiátrica, o que conduz por sua vez ao absentismo. ${ }^{2}$

A patologia somática, especialmente a osteoarticular ou musculosquelética, tem vindo a ser relacionada com o absentismo laboral prolongado, , ${ }^{3,12,17-19}$ tal como acontece no nosso estudo, em que verificamos existir uma associação positiva e estatisticamente significativa entre CIT prolongada e presença de patologia osteoarticular, oncológica e história cirúrgica recente.

Os resultados do estudo InCIT trazem informação importante para a prática clínica, sendo um estudo pioneiro nos cuidados de saúde primários em Portugal. O empa-
QUADRO II. Associação entre as diferentes variáveis sociodemo-

gráficas, profissionais, familiares e patológicas e a possibilidade de estar com CIT prolongada (OR e respectivos IC 95\%).

\begin{tabular}{|c|c|}
\hline & OR* (IC 95\%) \\
\hline \multicolumn{2}{|l|}{ Género } \\
\hline Masculino & 1 \\
\hline Feminino & $1,10(0,53-2,27)$ \\
\hline \multicolumn{2}{|l|}{ Idade (anos) } \\
\hline $18-30$ & 1 \\
\hline $31-50$ & $1,19(0,32-4,41)$ \\
\hline $51-65$ & $2,94(0,75-11,57)$ \\
\hline \multicolumn{2}{|l|}{ Estado civil } \\
\hline Solteiro & 1 \\
\hline Casado/união de facto & $0,34(0,09-1,26)$ \\
\hline Viúvo & $0,44(0,02-6,66)$ \\
\hline Divorciado/Separado & $0,60(0,12-2,99)$ \\
\hline \multicolumn{2}{|l|}{ Escolaridade (anos) } \\
\hline $0-4$ & 1 \\
\hline $5-6$ & $0,36(0,12-1,06)$ \\
\hline $7-9$ & $0,63(0,21-1,82)$ \\
\hline $10-12$ & $0,24(0,07-0,82)$ \\
\hline$>12$ & $0,07(0,01-0,36)$ \\
\hline \multicolumn{2}{|l|}{ Condição perante o trabalho } \\
\hline Trabalhador por conta própria & 1 \\
\hline Trabalhador por conta de outrem - setor público & $0,29(0,07-1,18)$ \\
\hline Trabalhador por conta de outrem - setor privado & $1,06(0,35-3,18)$ \\
\hline Desempregado & $0,37(0,11-1,29)$ \\
\hline Outra & $-(-)$ \\
\hline \multicolumn{2}{|l|}{ Tipo de trabalho } \\
\hline Colarinho branco & 1 \\
\hline Colarinho azul & $2,41(0,92-6,32)$ \\
\hline \multicolumn{2}{|l|}{ Escala de satisfação com o trabalho } \\
\hline Insatisfação & 1 \\
\hline Indiferença & $0,26(0,07-0,92)$ \\
\hline Satisfação & $0,34(0,12-1,00)$ \\
\hline \multicolumn{2}{|l|}{ Tipo de família } \\
\hline Nuclear & 1 \\
\hline Alargada & $1,62(0,63-4,20)$ \\
\hline Unitária & $3,52(0,68-18,34)$ \\
\hline Monoparental & $3,04(0,54-17,01)$ \\
\hline Reconstruída & $-(-)$ \\
\hline Outro & $-(-)$ \\
\hline \multicolumn{2}{|l|}{ APGAR familiar } \\
\hline $0-3$ & $-(-)$ \\
\hline $4-6$ & $-(-)$ \\
\hline $7-10$ & $-(-)$ \\
\hline
\end{tabular}




\begin{tabular}{|c|c|}
\hline & OR* (IC 95\%) \\
\hline \multicolumn{2}{|c|}{ Cirurgia recente } \\
\hline Não & 1 \\
\hline Sim & $14,49(3,83-54,80)$ \\
\hline \multicolumn{2}{|c|}{ Patologia osteoarticular } \\
\hline Não & 1 \\
\hline Sim & $6,14(2,79-13,48)$ \\
\hline \multicolumn{2}{|c|}{ Patologia oncológica } \\
\hline Não & 1 \\
\hline Sim & $4,62(1,18-18,08)$ \\
\hline \multicolumn{2}{|c|}{ Patologia psiquiátrica } \\
\hline Não & 1 \\
\hline Sim & $5,21(2,14-12,70)$ \\
\hline
\end{tabular}

*Ajustado para género, idade (contínua) e escolaridade (contínua) duos estudados em relação aos fatores avaliados e, desta forma, seria interessante realizar um estudo semelhante em que estes indivíduos não fossem incluídos. Não obstante, apesar dos desempregados incluídos no grupo dos casos estarem atualmente sem emprego, encontravam-se com CIT, estando assim impossibilitados de regressar à vida laboral, integrando assim o grupo de população em idade ativa incapacitada para o trabalho. Outros estudos realizados anteriormente incluíram também indivíduos desempregados, por motivos semelhantes. ${ }^{20}$

No contexto económico atual torna-se essencial minimizar os custos associados ao absentismo laboral para a melhor gestão dos recursos em saúde. A evidência gerada por este trabalho pode auxiliar os médicos

relhamento de casos e controlos por lista de utentes de cada médico de família permitiu eliminar o potencial viés associado a diferenças entre os sete médicos no que diz respeito à maior ou menor acessibilidade para prescrição de um CIT. No entanto, este trabalho não é isento de limitações, sendo de referir que a amostra utilizada no estudo InCIT é referente às listas de sete médicos de família pertencentes ao ACES de Matosinhos de quatro áreas geográficas distintas (Matosinhos, Lavra, São Mamede Infesta e Senhora da Hora), pelo que os resultados obtidos podem estar influenciados por diferenças regionais e não ser representativos da população portuguesa. Além disso a recolha de dados, apesar de ter sido feita de forma sistematizada, foi baseada em informação obtida através de entrevista telefónica e registada no processo clínico, estando ambos os métodos sujeitos a viés de informação, bem como possibilidade de viés de memória em relação aos antecedentes patológicos dos indivíduos estudados. Mais se acrescenta que o facto da folha de recolha de dados ter sido elaborada pelas investigadoras e não baseada totalmente em instrumentos previamente testados poderá comprometer a validade interna do estudo, não invalidando porém a aplicabilidade dos resultados obtidos.

A inclusão de indivíduos atualmente desempregados poderá ter aumentado a heterogeneidade dos indiví- de família a conhecer os indivíduos mais vulneráveis ao absentismo laboral prolongado, compreender os vários aspetos envolvidos e delinear estratégias preventivas, podendo ser também de grande utilidade para outras entidades na adoção de medidas preventivas centradas no local de trabalho. ${ }^{14,16}$

A consulta médica precoce e a abordagem holística do paciente, tão característica da Medicina Geral e Familiar, serão um instrumento eficaz na diminuição do absentismo laboral. Esta visão vem corroborar a necessidade de reconhecer precocemente e atuar nos indivíduos em maior risco de absentismo laboral..$^{12}$ Um utente com CIT prolongado terá um número aumentado de contatos com o seu médico de família e a incapacidade para o trabalho tem impacto na sua vida pessoal, estabilidade emocional e a nível da sua dinâmica familiar. ${ }^{21-23}$ Cabe ao médico de família o papel de capacitação dos indivíduos, bem como contribuir para o estreitar da barreira da reintegração, de forma a acelerar o regresso ao trabalho e diminuindo assim o impacto pessoal, familiar e social, associados ao absentismo laboral. ${ }^{22-24}$ Os dados obtidos neste estudo tornam possível o reconhecimento das características principais dos indivíduos para os quais devem ser dirigidas de medidas preventivas, custo-efetivas, que permitam redução de custos e do impacto pessoal, familiar e social, associados ao absentismo laboral. 


\section{REFERÊNCIAS BIBLIOGRÁFICAS}

1. Bekker MH, Rutte CG, van Rijswijk K. Sickness absence: A gender-focused review. Psychol Health Med 2009 Aug; 14 (4): 405-18.

2. Foss L, Gravseth HM, Kristensen P, Claussen B, Mehlum IS, Skyberg K. Risk factors for long-term absence due to psychiatric sickness: a register-based 5-year follow-up from the Oslo health study. J Occup Environ Med 2010 Jul; 52 (7): 698-705.

3. Graça L. Baixa por doença e incapacidade temporária para o trabalho. Lisboa: Observatório Português dos Sistemas de Saúde; 2002.

4. Labriola M, Lund T, Burr H. Prospective study of physical and psychosocial risk factors for sickness absence. Occup Med (Lond) 2006 Oct; 56 (7): 469-74.

5. Spierdijk L, van Lomwel G, Peppelman W. The determinants of sick leave durations of Dutch self-employed. J Health Econ 2009 Dec; 28 (6): 1185-96.

6. Bratberg E, Gjesdal S, Maeland JG. Sickness absence with psychiatric diagnoses: individual and contextual predictors of permanent disability. Health Place 2009 Mar; 15 (1): 308-14.

7. Laaksonen M, Piha K, Rahkonen O, Martikainen P, Lahelma E. Explaining occupational class differences in sickness absence: results from middle-aged municipal employees. J Epidemiol Community Health 2010 Sep; 64 (9): 802-7.

8. Agapito S, Cardoso de Sousa F.A influência da satisfação profissional no absentismo laboral. Rev Port Saúde Pública 2010 jul-dez; 28 (2): 132-9.

9. Decreto-Lei n. ${ }^{\circ} 28 / 2004$, de 4 de fevereiro. Diário da Republica n ${ }^{\circ} 29$ - I Série.

10. Agostinho M, Rebelo L. Família: do conceito aos meios de comunicação. Rev Port Saúde Pública 1988; 5: 18-21.

11. Siqueira M. Medidas do comportamento organizacional: ferramentas de diagnóstico e de gestão. Porto Alegre: Artmed; 2008.

12. Hoedeman R, Blankenstein AH, Krol B, Koopmans PC, Groothoff JW. The contribution of high levels of somatic symptom severity to sickness absence duration, disability and discharge. J Occup Rehabil 2010 Jun; 20 (2): 264-73.

13. Löve J, Holmgren K, Torén K, Hensing G. Can work ability explain the social gradient in sickness absence: a study of a general population in Sweden. BMC Public Health 2012 Mar; 12: 163.

14. Melchior M, Krieger N, Kawachi I, Berkman LF, Niedhammer I, Goldberg M. Work factors and occupational class disparities in sickness absence: findings from the GAZEL cohort study. Am J Public Health 2005 Jul; 95 (7): 1206-12.

15. Hensing G, Wahlström R. Swedish Council on Technology Assessment in Health Care (SBU). Chapter 7. Sickness absence and psychiatric disorders. Scand J Public Health Suppl 2004; 63: 152-80.

16. Hussey L, Turner S, Thorley K, McNamee R, Agius R. Work-related sickness absence as reported by UK general practitioners. Occup Med (Lond) 2012 Mar; 62 (2): 105-11.
17. Karels CH, Bierma-Zeinstra SM, Verhagen AP, Koes BW, Burdorf A. Sickness absence in patients with arm, neck and shoulder complaints presenting in physical therapy practice: 6 months follow-up. Man Ther 2010 Oct; 15 (5): 476-81.

18. Morris J, Watson PJ. Investigating decisions to absent from work with low back pain: a study combining patient and GP factors. Eur J Pain 2011 Mar; 15 (3): 278-85.

19. Linaker C, Harris EC, Cooper C, Coggon D, Palmer KT. The burden of sickness absence from musculoskeletal causes in Great Britain. Occup Med (Lond) 2011 Oct; 61 (7): 458-64.

20. Hultin H, Lindholm C, Möller J. Is there an association between longterm sick leave and disability pension and unemployment beyond the effect of health status? A cohort study. PLoS One 2012; 7 (4): e35614.

21. Swedish Council on Technology Assessment in Health Care. Sickness absence: causes, consequences, and physicians' sickness certification practice: a systematic literature review by the Swedish Council on Technology Assessment in Health Care (SBU). Scand J Public Health Suppl 2004; 63: 3-263.

22. Bryngelson A. Long-term sickness absence and social exclusion. Scand J Public Health 2009 Nov; 37 (8): 839-45.

23. Winde LD, Alexanderson K, Carlsen B, Kjeldgard L, Wilteus AL, Gjesdal S. General practitioners' experiences with sickness certification: a comparison of survey data from Sweden and Norway. BMC Fam Pract 2012 Mar 1; 13: 10.

24. Fundação Europeia para a Melhoria das Condições de Vida e de Trabatho. A Prevenção do absentismo no trabalho - sinopse da investigação. 1997. Disponível em http://www.eurofound.europa.eu/pubdocs/1997/15/pt/1/ef9715pt.pdf [acedido em 20/12/2012].

\section{CONFLITO DE INTERESSES E FINANCIAMENTO}

As autoras declaram não possuir qualquer tipo de conflito de interesses e não terem recebido qualquer tipo de financiamento externo.

\section{ENDEREÇO PARA CORRESPONDÊNCIA}

Joana Costa Gomes

Rua da Lagoa, s/número

4460-352 Senhora da Hora - Matosinhos

E-mail: joanacostabgomes@hotmail.com

Recebido em 26/12/2012

Aceite para publicação em 06/05/2013

Artigo escrito ao abrigo do novo acordo ortográfico. 


\section{ABSTRACT}

\section{DETERMINANTS OF LONG-TERM WORK ABSENTEEISM - THE INCIT STUDY}

Goal: The aim of this study was to evaluate the determinants of long-term absenteeism from work including socio-demographic, work-related and disease-related characteristics.

Study design: Prospective case-control study

Setting: Unidade Local de Saúde de Matosinhos

Study population: Individuals between 18 and 65 years of age were considered cases if they were absent from work for a period longer than 30 consecutive days. For each case, a control was selected randomly from the list of the same family physician.

Material and methods: Information was gathered regarding socio-demographic and disease-related characteristics (recent surgery, psychiatric, musculoskeletal and neoplastic disease) of cases and controls. The Family APGAR and the Work Satisfaction Scale were used to evaluate family function and work satisfaction. Odds ratios and $95 \%$ confidence intervals, adjusted for gender, age and education, were computed to estimate the association between these variables and long-term work absenteeism. Results: During the study period, 76 cases were identified and 76 controls were selected. About $56 \%$ of cases and $62 \%$ of controls were men. This difference was not significant. Mean age was higher among the cases (50.0 versus $42.5 ; \mathrm{p}<0.001)$ and cases were less educated $(p<0.001)$. A higher proportion of cases had blue-collar occupations $(p=0.001)$ and cases had lower family APGAR scores $(p=0.008)$. There was higher risk of long-term absenteeism for individuals with musculoskeletal disease (OR 6.14; $95 \% \mathrm{Cl} 2.79$ - 13.48), neoplastic disease (OR 4.62; 95\% Cl 1.18-18.08), psychiatric disease (OR 5.21; $95 \% \mathrm{Cl} 2.14$ - 12.70) and recent surgery (OR 14.49; $95 \% \mathrm{Cl} 3.83-54.80$ ).

Conclusion: This study may help clinicians to recognize individuals at higher risk of long-term work absenteeism and enable them to apply cost-effective preventive measures to decrease the health, family and social burdens of this problem.

Key words: Absenteeism; Epidemiologic Factors; Case-control Study. 